\title{
Revisión
}

\section{Hiperandrogenismo: pubarquia precoz y síndrome de ovario poliquístico. Etiología y posibilidades terapéuticas}

\author{
L. García Blanco, C. Azcona San Julián \\ Servicio de Pediatría, Unidad de Endocrinología Pediátrica. \\ Clínica Universidad de Navarra. Pamplona. España.
}

Palabras clave: - Hiperandrogenismo - Ovario poliquístico - Pubarquia
La síntesis de andrógenos en la mujer se produce en las glándulas suprarrenales, en el ovario y en los tejidos diana periféricos para la acción androgénica (piel, músculo y tejido adiposo).

El exceso de producción androgénica (o hiperandrogenismo) en la infancia y adolescencia puede determinar alteraciones en estos tejidos diana, condicionando la aparición de hirsutismo, acné, trastornos menstruales o virilización, cuya intensidad y momento de aparición dependerán del origen del exceso de producción de andrógenos.

La etiología del hiperandrogenismo variará según la edad. Así, en el periodo prepuberal, en ausencia de maduración gonadal la hiperproducción androgénica tendrá origen en la glándula suprarrenal en la mayoría de los casos, mientras que en el periodo peripuberal y postpuberal el ovario será el responsable.

\section{Hyperandrogenism: precocious pubarche and polycystic ovary syndrome. Etiology and therapeutic possibilities}

The synthesis of androgens in women is produced in the adrenal gland, ovary, and peripheral target tissues for androgen action (skin, muscle and adipose tissue).

Excessive androgen production (or hyperandrogenism) in childhood and adolescence may determine alterations in these target tissues, determining the onset of hirsutism, acne, menstrual disorders or virilization, whose intensity and time of onset depend on the origin of the excess production of androgens.

Key words:

- Hyperandrogenism

The etiology of hyperandrogenism will vary according to age. Therefore, in the prepubertal period in the absence of androgen overproduction gonadal maturation have originated in the adrenal gland in - Policystic ovary

- Pubarche most cases, while in the peripubertal post-pubertal period the ovary is the main cause.

\section{INTRODUCCIÓN}

\section{Conceptos}

- Hirsutismo: exceso de crecimiento de vello corporal en la mujer en zonas consideradas típicamente androgénicas: zona supralabial, mentón, pabellones auriculares, tórax, abdomen, espalda, glúteos, y cara interior y anterior de los muslos. El hirsutismo se debe a un exceso de producción de andrógenos o a un aumento del metabolismo cutáneo de los andrógenos. El aumento de la actividad androgénica en las glándulas sebáceas y apocrinas puede determinar la aparición del acné vulgar.

- Hipertricosis: es el aumento de pelo corporal en zonas donde ya existe normalmente en la mujer, sobretodo en antebrazos y piernas, y menos frecuentemente en muslos y tronco. Se da por fac- 
tores raciales y familiares, o secundariamente al uso continuado de determinados fármacos ${ }^{1}$.

\section{Causas de hiperandrogenismo en la infancia y adolescencia (Tabla 1)}

\section{Causas más frecuentes del hiperandrogenismo en el periodo prepuberal}

\section{Pubarquia precoz}

Es la presentación clínica más frecuente de hiperandrogenismo en el periodo prepuberal. Se define como la aparición de vello pubiano antes de los ocho años en las niñas y de los nueve años en los niños, en ausencia de otros signos de actividad gonadal. Es mucho más frecuente en el sexo femenino.

La mayoría de los casos se deben a un aumento prematuro de la secreción de andrógenos suprarrenales. Los niveles de estas hormonas, específicamente del sulfato de dehidroepiandrosterona (DHEAS), que es el marcador del proceso de adrenarquia, se encuentran discretamente elevados para la edad cronológica.

En estas pacientes se observa una aceleración moderada y transitoria de la velocidad de crecimiento y de la maduración ósea, por lo que la mayoría presenta al diagnóstico una talla alta, pero adecuada en general, a su edad ósea².

En el 7-10\% de los casos, la pubarquia precoz puede ser la primera manifestación clínica de una forma no clásica de hiperplasia suprarrenal congénita por déficit de 21-hidroxilasa. En estos casos, la aparición de vello pubiano se acompaña en general de signos de virilización, de una aceleración progresiva de la velocidad de crecimiento, y de una edad ósea dos o más años por delante de la edad cronológica.

Sin embargo, en ocasiones, el cuadro clínico no es tan Ilamativo. Por este motivo, es obligado descartar esta entidad mediante un test de estimulación con hormona adrenocorticotropa (ACTH) cuando las cifras basales de 17-hidroxiprogesterona (17OHP) plasmática sean superiores a $3 \mathrm{nmol} / \mathrm{l}$ (100 ng/dl). Se establecerá el diagnóstico de sospecha de hiperplasia suprarrenal congénita cuando los niveles de 17-OHP a los 60 minutos de la administración de ACTH sean superiores a 1500 ng/dl. Las respuestas entre 1000-1500 ng/dl parecen corresponder a portadores (heterozigotos para la mutación) de esta forma no clásica; el diagnóstico definitivo precisa el estudio del gen CYP-21B, que codifica la enzima 21-hidroxilasa ${ }^{3}$.

\section{Tabla 1. Causas de hiperandrogenismo en la infancia y adolescencia}

1. Hiperandrogenismo suprarrenal funcional:

a) Primario: pubarquia precoz secundaria a adrenarquia prematura

b) Formas tardías de hiperplasia suprarrenal congénita:

- Déficit de 21-hidroxilasa

- Déficit de 3 ß-hidroxiesteroide-deshidrogenasa

- Déficit de 11 ß-hidroxilasa

c) Sindrome de Cushing

d) Hiperprolactinemia

2. Hiperandrogenismo ovárico funcional:

a) Síndrome del ovario poliquístico primario

b) Síndrome del ovario poliquístico secundario:

- Enfermedades suprarrenales virilizantes

- Bloqueos de la esteroidogénesis ovárica

- Síndromes de insulinorresistencia

3. Hiperproducción periférica de andrógenos:

a) Obesidad

b) Hiperandrogenismo idiopático (producción glandular normal de andrógenos)

4. Hiperandrogenismo tumoral

5. latrogenia: administración exógena de derivados androgénicos 
La pubarquia precoz aislada no afecta al desarrollo puberal ni a la talla final. En la mayoría de estas niñas, la talla final se encuentra discretamente por encima de la talla diana, siguiendo la tendencia secular de la población. Sin embargo, una de cada dos pacientes con pubarquia precoz desarrolla un cuadro de hiperandrogenismo ovárico -o síndrome del ovario poliquístico (SOP) - en la adolescencia, caracterizado por la asociación de hirsutismo y trastornos menstruales, y acompañado de hiperinsulinismo y de un perfil lipídico aterogénico, aun en ausencia de obesidad.

Esta entidad constituye un factor de riesgo para el desarrollo de diabetes tipo 2 y enfermedad cardiovascular. El hiperinsulinismo y las alteraciones lipídicas son ya detectables en el periodo prepuberal y a lo largo de toda la pubertad.

Aunque las manifestaciones clínicas del hiperandrogenismo no suelen aparecer hasta transcurridos tres o más años desde la menarquia, las adolescentes con pubarquia precoz presentan ya durante la pubertad una síntesis de andrógenos ováricos exagerada y disfunción ovulatoria en presencia de ciclos menstruales a parentemente regulares ${ }^{4}$.

Estas alteraciones endocrino-metabólicas son mucho más frecuentes en las niñas con pubarquia precoz que tuvieron un peso bajo al nacer para la edad gestacional y que, a su vez, son las que presentan un hiperinsulinismo más marcado, sobre todo después de la menarquia. La administración de agentes sensibilizantes de la insulina durante la adolescencia es capaz de normalizar el cuadro biológico, específicamente, el hiperandrogenismo y el hiperinsulinismo, y de mejorar el perfil lipídico, lo que confirma el papel clave de la insulina en el desarrollo de estas alteraciones.

Las niñas con bajo peso al nacer que desarrollan pubarquia precoz presentan con mayor frecuencia pubertad adelantada rápidamente evolutiva; el tratamiento con metformina de inicio prepuberal mejora el perfil endocrino-metabólico, y el patrón de distribución de grasa corporal con efectos sostenidos después de su suspensión consigue normalizar el inicio y la progresión de la pubertad, la edad de la menarquia, y muy probablemente, la talla final ${ }^{5}$.

Por tanto, la pubarquia precoz no puede considerarse una variante normal, fundamentalmente cuando existen antecedentes de peso bajo al nacer. Es aconsejable un seguimiento continuado de estas pacientes, al menos hasta tres años después de finalizada la pubertad.

En el varón, la pubarquia precoz no se asocia a alteraciones endocrino-metabólicas ni a un peso bajo al nacer ${ }^{6}$.

\section{Causas más frecuentes del hiperandrogenismo en la adolescencia (Fig. 2 y Tabla 2)}

\section{Hiperandrogenismo ovárico}

El SOP es la causa más frecuente de hiperandrogenismo de comienzo peripuberal (3\% de la población femenina). La definición más aceptada es la de los National Institutes of Health $(\mathrm{NIH})$, que requiere para el diagnóstico la existencia de hiperandrogenismo y de anovulación crónica, pero no la presencia de ovarios poliquísticos, después de excluir otros factores. Se acompaña con frecuencia, aun en ausencia de obesidad, de resistencia a la insulina, de un perfil lipídico aterogénico y de un aumento de grasa de disposición central.

El SOP es un estado proinflamatorio, de prediabetes tipo 2 y preenfermedad cardiovascular, y por tanto podría considerarse parte del denominado síndrome metabólico o síndrome $X^{7}$.

Las pacientes con SOP tienen mayor riesgo de desarrollar precozmente diabetes tipo 2 y enfermedad cardiovascular; este riesgo está en relación directa con el grado de hiperinsulinismo e insulinorresistencia, y es más elevado si existe obesidad.

\section{Grupos de riesgo para el desarrollo de SOP}

Entre las entidades de riesgo para el desarrollo de SOP hay que señalar: 1) la obesidad de inicio precoz asociada a resistencia a la insulina; 2 ) el bajo peso al nacer asociado a una recuperación postnatal rápida y exagerada de peso y al desarrollo de 


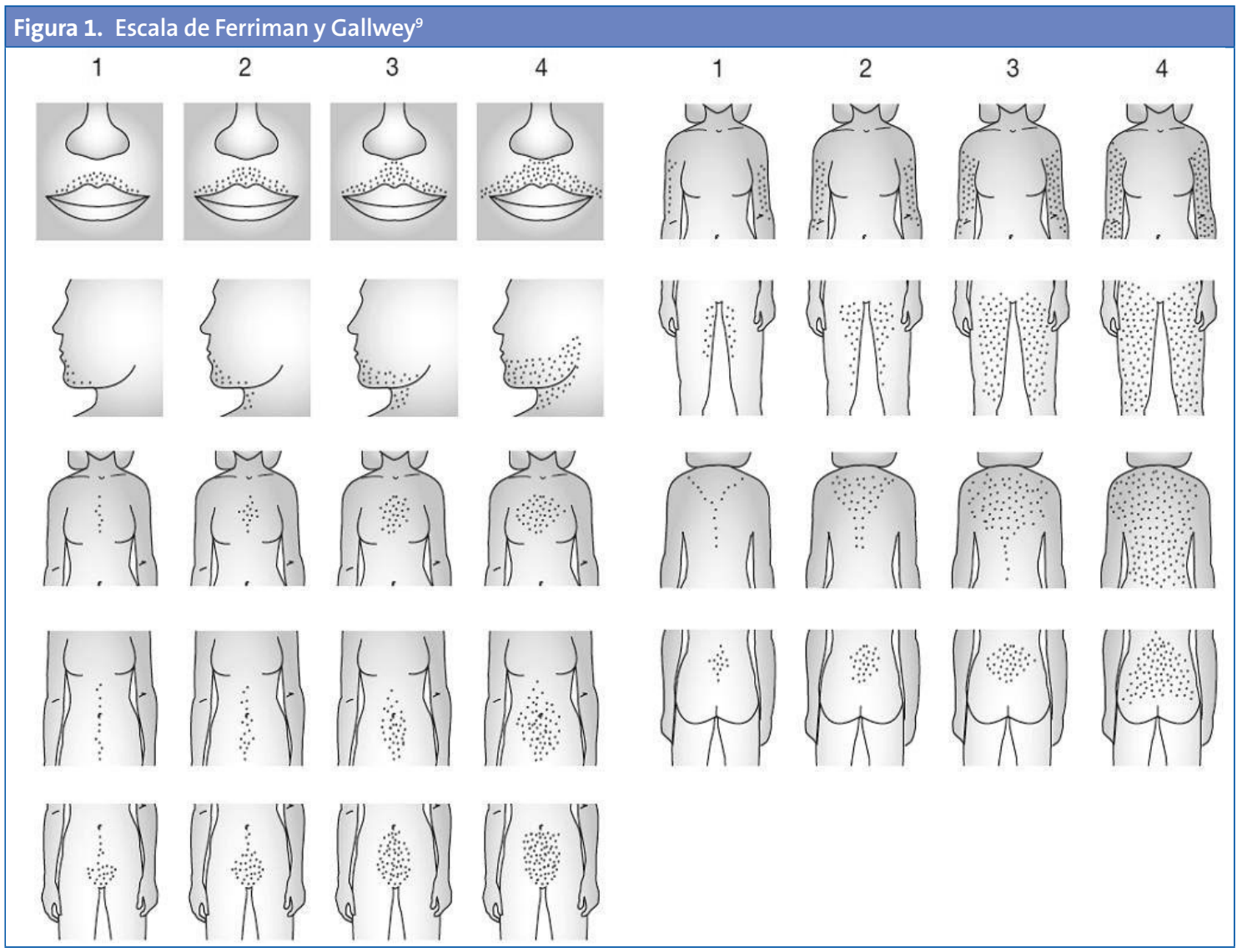

pubarquia precoz (aparición de vello pubiano antes de los ocho años), y/o pubertad adelantada e hiperinsulinismo ${ }^{8}$; 3) la prolongación del periodo de anovulación fisiológica mas allá de dos años postmenarquia y 4) la hiperplasia suprarrenal congénita y otros trastornos virilizantes.

\section{Presentación clínica del SOP}

La combinación de hirsutismo moderado de progresión lenta, asociado a irregularidades menstruales, acné, acantosis nigricans (áreas de pigmentación cutánea en cuello y axilas), obesidad y/o exceso de adiposidad central es la forma de presentación clínica más común del SOP.

En el examen físico se constatará la existencia y el grado de hirsutismo, de acuerdo con la escala de Ferriman y Gallwey que se presenta en la Fig. ${ }^{19}$.

Una puntuación superior a 8 es diagnóstica de hirsutismo, pero en el SOP dicha puntuación suele estar alrededor de 12-15; una puntuación superior a 30 es sospechosa de tumor secretor de andrógenos (o de administración de derivados androgénicos), y suele acompañarse de otros signos de virilización, como clitoromegalia, alopecia y aumento de la masa muscular. Un cociente cintura cadera elevado $(\geq 0,8)$ sugiere la existencia de un exceso de adiposidad central; la presencia de acantosis nigricans se asocia a resistencia a la insulina ${ }^{10}$.

\section{Hiperandrogenismo de origen periférico}

La obesidad puede causar hiperandrogenemia y amenorrea, suele ser común la existencia de acantosis nigricans y de insulinorresistencia.

\section{Hiperandrogenismo suprarrenal funcional}

Suelen presentar hirsutismo y con menor frecuencia anomalías menstruales. 
Figura 2. Evaluación inicial y diagnóstico diferencial del hirsutismo ${ }^{1}$

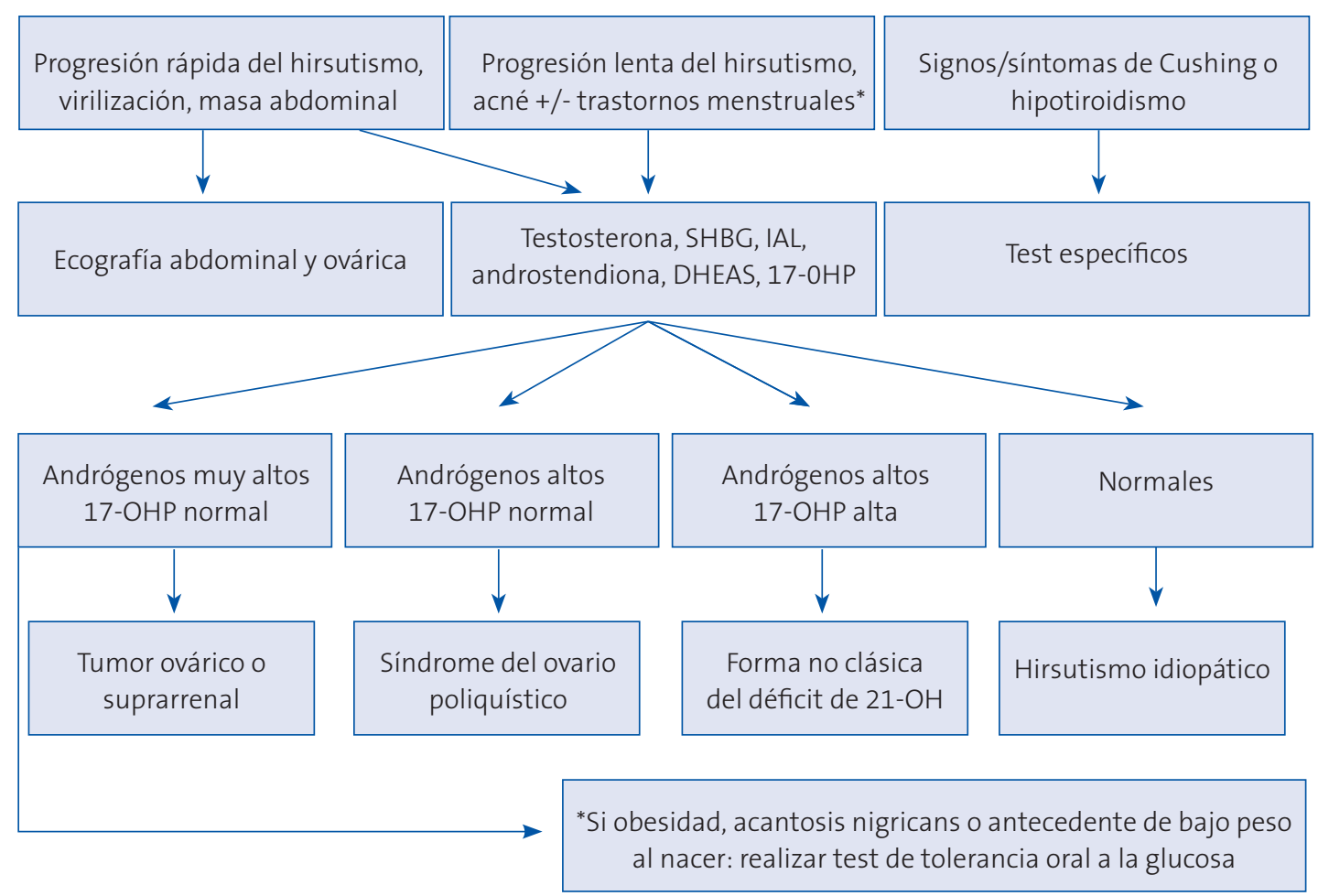

17-OHP: 17-hidroxiprogesterona; DHEAS: sulfato de dehidroepiandrosterona; 21-OH: 21-hidroxilasa; SHBG: sex hormone-binding globulin; IAL: índice de andógenos libres.

\section{Hiperprolactinemia}

Se asocia a hirsutismo, acné y amenorrea. El 50\% presentan también galactorrea.

\section{TRATAMIENTO}

\section{Sintomático}

En adolescentes con sobrepeso $u$ obesidad, la dieta y el ejercicio físico constituyen los pilares fundamentales del tratamiento, tanto para normalizar la ciclicidad menstrual como para evitar complicaciones a largo plazo como la diabetes tipo 2.

\section{Cosmético}

Las medidas cosméticas para eliminar el vello, como la depilación eléctrica o el láser, están indicadas en el hirsutismo idiopático, y pueden ser de utilidad en las fases iniciales del tratamiento en el SOP con hirsutismo moderado o severo, ya que la terapéutica farmacológica no es efectiva sobre el vello corporal hasta transcurridos 6-12 meses.

\section{Tratamiento farmacológico}

Tiene como objetivo no solo mejorar los síntomas derivados del exceso de andrógenos, sino también modificar los marcadores de riesgo cardiovascular que pueden favorecer las complicaciones a medio y largo plazo. Los anticonceptivos orales (ACO) mejoran la sintomatología y regularizan los ciclos menstruales, pero administrados en monoterapia agravan la resistencia a la insulina y la adiposidad corporal, e incrementan el riesgo cardiovascular. El tratamiento combinado con un sensibilizante de la insulina (metformina, $850 \mathrm{mg} /$ día) y un antiandrógeno puro (flutamida, $62,5 \mathrm{mg} / \mathrm{día}$ ) ${ }^{12}$ en dosis bajas 
Tabla 2. Pruebas utilizadas en el diagnóstico diferencial del hirsutismo ${ }^{11}$

\begin{tabular}{|c|c|c|c|}
\hline Nombre & Determinaciones & Parámetros & Valoración \\
\hline ACTH (Synachtén $\left.{ }^{\circledast}\right): 0,25$ mg, IV, 1 dosis & Basal y 60 minutos & 17-OHP, cortisol & $\begin{array}{l}17-\mathrm{OHP}>1000 \mathrm{ng} / \mathrm{dl}^{*} \\
17-\mathrm{OHP}>368 \mathrm{ng} /\left.\mathrm{dl}\right|^{* *}\end{array}$ \\
\hline $\begin{array}{l}\left.\text { Acetato de leuprorelina (Procrin }{ }^{\circledR}\right): 500 \mu \mathrm{g}, \mathrm{SC} \text {, } \\
1 \text { dosis }\end{array}$ & Basal, 3 horas y 24 horas & $\begin{array}{l}\mathrm{LH}, \mathrm{FSH}, 17-\mathrm{OHP} \text {, } \\
\text { estradiol }\end{array}$ & $\begin{array}{l}\text { Hiperrespuesta*** } \\
17-\mathrm{OHP}>160 \mathrm{ng} / \mathrm{dl}\end{array}$ \\
\hline $\begin{array}{l}\text { Test de tolerancia oral a la glucosa: 1,75 g/kg, } \\
\text { máximo } 75 \mathrm{~g}\end{array}$ & $\begin{array}{l}\text { Basal, } 30,60 \text {, y } 120 \\
\text { minutos }\end{array}$ & Glucosa, insulina & $\begin{array}{l}\text { Hiperinsulismo: pico de } \\
\text { insulina }>150 \mathrm{mU} / \mathrm{l}\end{array}$ \\
\hline
\end{tabular}

17-OHP: 17-hidroxiprogesterona; ACTH: hormona adrenocorticotropa; FSH: hormona folículo estimulante; IV: vía intravenosa;

LH: hormona luteinizante; SC: vía subcutánea.

* Sospecha de forma no clásica de hiperplasia suprarrenal congénita por déficit de 21-hidroxilasa. El diagnóstico definitivo requiere confirmación molecular.

**Valores superiores al promedio +2 desviaciones estándar (DS) de los valores en controles; hiperrespuesta suprarrenal o hiperandrogenismo suprarrenal funcional.

***Valores superiores al promedio + 2 DS de los valores en controles; sugestivos de hiperandrogenismo ovárico funcional.

no solo normaliza la hipersecreción de andrógenos y los síntomas clínicos, incluida la frecuencia ovulatoria, sino que también mejora el estado metabólico, la composición corporal y los marcadores de riesgo que acompañan al hiperandrogenismo ovárico; por ejemplo, los parámetros de inflamación (IL-6, neutrofilia relativa, y proteína C reactiva ultrasensible), aumentando los niveles de adiponectina. La mejoría del hiperinsulinismo podría mediar estas modificaciones y, por tanto, disminuir el riesgo de enfermedad cardiovascular y de diabetes tipo 2 . La flutamida es un bloqueante del receptor de andrógenos que carece de actividad progestágena, estrogénica, corticoidea o antigonadotrópica ${ }^{13}$.
Durante mucho tiempo, se ha considerado que la flutamida podía ser hepatotóxica. Existen datos que confirman que esta potencial hepatotoxicidad es dosis-dependiente y no idiosincrásica. En dosis de $125 \mathrm{mg} /$ día o inferiores, no se ha reportado ni un solo caso de aumento de las transaminasas en series que incluían mas de 900 pacientes en tratamientos de hasta seis años de duración. Recientemente, se ha reportado que la adición de dosis bajas de pioglitazona (un agonista PPARg) al tratamiento combinado con metformina y flutamida consigue normalizar aún mas los parámetros endocrinometabólicos, y además disminuye la proporción de grasa visceral y el grosor de la íntima

Figura 3. Evaluación inicial y diagnóstico diferencial del hirsutismo ${ }^{1}$

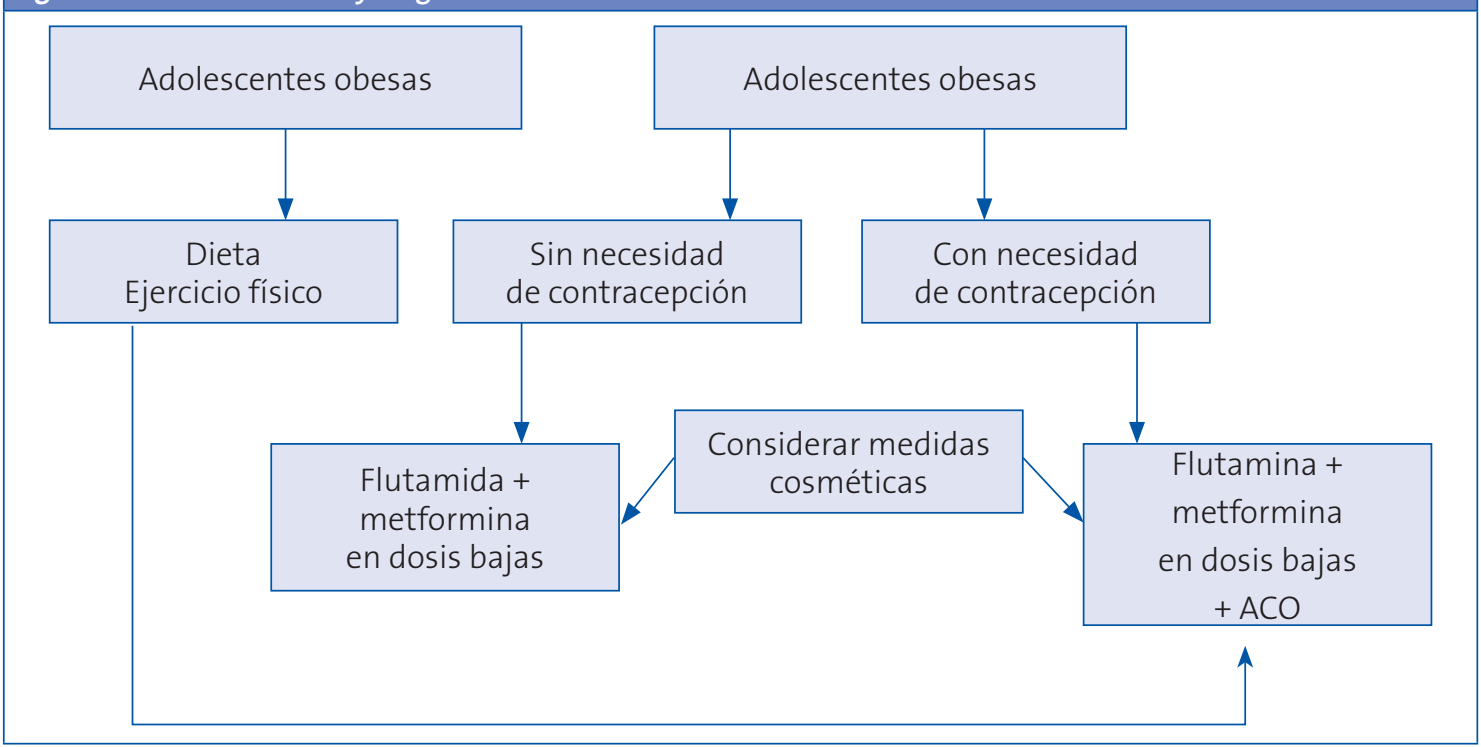


carotídea, que se encuentra aumentada en estas pacientes y que constituye un factor adicional de riesgo cardiovascular.

La adición de un ACO que contenga drospirenona al tratamiento combinado con flutamida y metformina en el caso de que exista riesgo de embarazo no modifica los efectos beneficiosos de la terapéutica combinada sobre los marcadores de riesgo cardiovascular.

Se recomienda una duración mínima del tratamiento de dos años. Sin embargo, el periodo de remisión obtenido al suspender el tratamiento es muy variable y se precisa un mayor número de estudios prospectivos para llegar a un consenso. Las recomendaciones terapéuticas se resumen en la Fig. $3^{14}$. El tratamiento preventivo con metformina

\section{BIBLIOGRAFÍA}

1. Ibáñez L, Marcos MV, Díaz R. Hirsutismo y trastornos menstruales en la adolescencia. An Pediatr Contin. 2009;7:144-51.

2. Idkowiak J, Lavery GG, Dhir V, Barrett TG, Stewart PM, Krone N, et al. Premature adrenarche: novel lessons from early onset androgen excess. Eur J Endocrinol. 2011;165(2):189-207.

3. Armengaud JB, Charkaluk ML, Trivin C, Tardy V, Breart $G$, Brauner R, et al. Precocious Pubarche: distinguishing late onset congenital adrenal hyperplasia from premature adrenarche. J Clin Endocrinol Metab. 2010;94:2835-40.

4. Oberfield SE, Sopher AB, Gerken AT. Approach to the girl with early onset of pubic hair. J Clin Endocrinol Metab. 2011;96(6):1610-22.

5. Ibáñez L, Potau N, Dunger D, de Zegher F. Precocious pubarche in girls and the development of androgen excess. J Pediatr Endocrinol Metab. 2000;13(Suppl 5):1261-3.

6. Laron Z (ed.). European Conference. Diagnosis and Treatment of Central Precocious Puberty: State of The Art 1999. J Ped Endocrinol Metab. 2000;13:723-38.

7. Weiss R, Dziura J, Burgert TS, Tambolarne WV, Taksali SE, Yyeckel CW, et al. Obesity and the metabolic syndrome in children and adolescents. N Engl J Med. 2004;350:2362-74.

8. Bridges NA, Cooke A, Healy MJ, Hindmarsh PC, Brook CG. Standards for ovarian volume in childhood and puberty. Fertil Steril. 1993;60(3):456-60. en pacientes de riesgo para el desarrollo de SOP es efectivo si se inicia antes de la pubertad, por lo que el periodo peripuberal podría constituir una ventana adecuada de intervención en estas pacientes ${ }^{15}$ (Fig. 3).

\section{CONFLICTO DE INTERESES}

Los autores declaran no presentar conflictos de intereses en relación con la redacción y publicación del presente estudio.

\section{ABREVIATURAS}

17-OHP: 17-hidroxiprogesterona - ACO: anticonceptivos orales $\bullet$ ACTH: hormona adrenocorticotropa $\bullet$ DHEAS: dehidroepiandrosterona $\bullet$ DS: desviaciones estándar $\bullet \mathrm{NIH}: \mathrm{Na}$ tional Institutes of Health • SOP: síndrome del ovario poliquístico.

9. Ferriman D, Gallway JD. Clinical assessment of body hair growth in women. J Clin Endocrinol Metab. 1961;21:1440-7.

10. Ibáñez L, Díaz R, López-Bermejo A, Marcos MV. Clinical spectrum of premature pubarche: Links to metabolic syndrome and ovarian hyperandrogenism. Rev Endocr Metab Disord. 2009;10:63-76.

11. Argente J, Carrascosa L, Gracia R, Rodríguez H. Hiperandrogenismo. En: Tratado de Endocrinología Pediátrica y de la adolescencia, 2. a ed. Madrid: Doyma; 2000. p. 1043-61.

12. Ibáñez L, de Zegher F. Low-dose flutamide-metformin therapy for hyperinsulinemic hyperandrogenism in non-obese adolescents and women. Hum Reprod Update. 2006;12:243-52.

13. Paradisi R, Venturoli S. Retrospective observational study on the effects and tolerability of flutamide in a large population of patients with various kinds of hirsutism over a 15-yearperiod. Eur J Endocrinol. 2010;163(1):139-47.

14. López-Bermejo A, Díaz M, Moran E, de Zegher F, Ibañez L. A single nucleotide polymorphism in STK11 influences insulin sensitivity and metformin efficacy in hyperinsulinemic girls with androgen excess. Diabetes Care. 2010;33(7):1544-8.

15. Ibáñez L, López-Bermejo A, Díaz M, Marcos MV, de Zegher F. Metformin treatment for 4 yr to reduce total and visceral fat in low-birthweight girls with precocious pubarche. J Clin Endocrinol Metab. 2008; 93:1841-5. 